\title{
Quality of life and healthcare utilisation in cystic fibrosis: a multicentre study
}

\author{
Judy M. Bradley*,\#, Steven W. Blume ", Maria-Magdalena Balp ${ }^{+}$, \\ David Honeybourne $^{\S}$ and J. Stuart Elborn ${ }^{\#, f}$
}

ABSTRACT: The aim of our study was to discover the health status and healthcare utilisation associated with pulmonary exacerbations in cystic fibrosis (CF) and chronic Pseudomonas aeruginosa infection.

Patients with CF from five UK CF centres attended two visits, 8-12 weeks apart. They were classified at visit 1 as being in one of the three health states: no current pulmonary exacerbation; "mild" (no hospitalisation) pulmonary exacerbation; and "severe" (hospitalisation) pulmonary exacerbation. All patients completed the Cystic Fibrosis Questionnaire-Revised (CFQ-R) and EuroQol (EQ-5D) and a clinical form, and forced expiratory volume in $1 \mathrm{~s}$ (FEV1) was measured at visits 1 and 2. Annual healthcare utilisation data were collected.

94 patients of mean \pm SD age $28.5 \pm 8.2$ yrs and $F E V_{1} 58.7 \pm 26.8 \%$ were recruited. 60 patients had no pulmonary exacerbation, 15 had a mild and 19 had a severe pulmonary exacerbation at visit 1. EQ-5D and CFQ-R data showed that the worse the exacerbation, the poorer the healthrelated quality of life (HRQoL). There were strong relationships between the CFQ-R and EQ-5D domain scores. The mean rate of pulmonary exacerbations per patient per year was 3.6 (1.5 in hospital and 2.2 at home). The mean length of stay per hospital pulmonary exacerbation was 9 days.

As exacerbation status worsens, patients experience worse HRQoL. There is a significant healthcare burden associated with treatment of pulmonary exacerbation and long-term prophylaxis.

KEYWORDS: Health utility, observational study

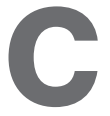
ystic fibrosis (CF) is one of the most common life-threatening autosomal recessive inherited diseases. Approximately one in every 2,500 children is born with CF and, although the average life expectancy of patients with $\mathrm{CF}$ is around $37 \mathrm{yrs}$, many patients die in their late teens or early 20 s due to recurrent respiratory infections causing progressive lung disease $[1,2]$. Patients are repeatedly infected by a range of opportunistic bacterial pathogens that are acquired in an age-dependent sequence; however, the most common pathogen in CF is Pseudomonas aeruginosa [3]. Once chronically infected with $P$. aeruginosa, there is a progressive decline in lung function with episodes of acute worsening of respiratory symptoms, usually referred to as pulmonary exacerbations [4]. Studies have shown that patients with frequent pulmonary exacerbations experience an accelerated decline in lung function and have a greater 3-yr risk of death or transplant $[4,5]$.
Antibiotic treatment targeted against the predominant pathogen is the mainstay of CF pulmonary exacerbation management and has contributed to a dramatic increase in life expectancy in the past few decades [6, 7]. Treatment of pulmonary exacerbations often includes intravenous antibiotic therapy, which can be delivered in hospital or at home. Home therapy may be appropriate for selected patients; however, the evidence for this is limited and not all studies have shown this to be a costeffective option [6, 7]. The use of inhaled antibiotics is focused on reducing the number of pulmonary exacerbations in CF. A number of landmark clinical trials have demonstrated the efficacy of inhaled antibiotics, for example, inhaled tobramycin solution, tobramycin inhalation powder and aztreonam lysine [8-11].

Recent studies have shown adoption of increasingly complex, but effective, drug therapies for the management of pulmonary exacerbations in $\mathrm{CF}$.

\section{AFFILIATIONS}

*Health and Rehabilitation Sciences Research Institute, School of Health Sciences, University of Ulster, Newtownabbey

"Dept of Respiratory Medicine, Belfast City Hospital, Belfast Health and Social Care Trust,

${ }^{f}$ Centre for Infection and Immunity, Queens University Belfast, Belfast, and

${ }^{\S}$ West Midlands Adult CF Centre, Birmingham Heartlands Hospital, Birmingham, UK.

- United BioSource Corporation, Bethesda, MD, USA.

${ }^{+}$Novartis Pharma, Basel, Switzerland

\section{CORRESPONDENCE}

J.M. Bradley

Health and Rehabilitation Sciences Research Institute, School of Health Sciences

University of Ulster

Newtownabbey BT37 OQB

UK

E-mail: jm.bradley@ulster.ac.uk

Received:

Dec 222011

Accepted after revision:

May 102012

First published online:

July 262012 
For example, data from the USA show that the use of inhaled antibiotics increased from $25.7 \%$ to $39.3 \%$ between 2001 and 2007 ( $\mathrm{p}=0.05)$, and treatments associated with P. aeruginosa infection substantially increase average annual expenditures across all age groups [12]. These changes have resulted in considerable economic costs with medical care costs of $\mathrm{CF}$ tripling in the last decade [12-14]. There are limited current data on specific healthcare utilisation associated with pulmonary exacerbations in UK.

Pulmonary exacerbations can have a negative impact on health-related quality of life (HRQoL) that lasts long after the pulmonary exacerbation has resolved $[15,16]$. The development and use of disease-specific quality of life instruments, such as the Cystic Fibrosis Questionnaire-Revised (CFQ-R), have increased our understanding of the link between pulmonary exacerbations and HRQoL by focusing on diseasespecific aspects of health status $[17,18]$. There is also little information in CF on the impact of pulmonary exacerbations on generic health status, e.g. using the EuroQol (EQ-5D). The EQ-5D provides a simple descriptive profile and a single index value of health status [19-21]. It can be used to calculate quality-adjusted life-years for future cost-effectiveness analyses of treatments, allowing comparison between therapies across different therapeutic areas. It is not currently known how these two questionnaires compare.

The aim of this study was to explore health status associated with pulmonary exacerbations in patients with CF and chronic $P$. aeruginosa infection. Additional objectives were to determine the relationship between CFQ-R and EQ-5D and to describe the healthcare utilisation associated with pulmonary exacerbations.

\section{MATERIAL AND METHODS Study subjects}

Adolescents (aged $>16$ yrs) and adults diagnosed with CF and chronic $P$. aeruginosa infection taking inhaled/oral antibiotics (tobramycin, colistin or azithromycin) were recruited. Patients were included if they were able to give written informed consent, complete the protocol requirements and if they were currently and normally resident in the UK. Patients were excluded if they had acute illness not related to a pulmonary exacerbation, cognitive or other impairment (e.g. visual) that would interfere with the study requirements. Patients were also excluded if they had a significant co-morbidity not related to $\mathrm{CF}$ that could influence health status, or were being considered for or on a lung transplantation list. This study received approval from local ethics committee (08NIR03/76).

\section{Study design}

The objectives of our study were to explore the health status associated with pulmonary exacerbations in patients with $\mathrm{CF}$ and chronic $P$. aeruginosa infection; to determine the relationship between EQ-5D and the CFQ-R; and to describe the healthcare utilisation associated with regular management of CF patients with $P$. aeruginosa infection and for pulmonary exacerbations. The study was conducted at five sites in England and Northern Ireland with a planned total enrolment of 100 participants. No formal sample size calculation was performed. It was planned that at least 30 patients in each of the pulmonary exacerbation status groups would be recruited at visit 1; however, this recruitment goal of 30 patients per pulmonary exacerbation status was not achieved due to the small numbers of CF patients coming to clinics with a pulmonary exacerbation in progress, whether mild or severe. After extending the enrolment time-period to try to obtain the target number of patients, and still not having any increase in the number of patient in these categories, it was determined to ease this goal and enrol patients with CF regardless of current pulmonary exacerbation status at visit 1 .

\section{Methods}

Patients attended two visits. Patients were classified at visit 1 as being in one of three health states: no current pulmonary exacerbation, "mild" pulmonary exacerbation (pulmonary exacerbation managed without the need for hospitalisation) and "severe" pulmonary exacerbation (pulmonary exacerbation requiring hospitalisation). There is no clear consensus on the definition of an acute pulmonary exacerbation [22]. For the purpose of this study, a pulmonary exacerbation was defined as the presence of two or more of the following criteria: decreased forced expiratory volume in $1 \mathrm{~s}$ (FEV1) of $\geqslant 10 \%$ from baseline (baseline defined as best in last 6 months); decreased weight of at $\geqslant 1 \mathrm{~kg}$ from baseline (baseline defined as best in last 6 months); change in sputum colour or increase in sputum production from normal; increased cough from normal; or decrease in energy from normal; and also a decision to commence new oral or i.v. antibiotics for treating a pulmonary exacerbation. The need for hospitalisation was driven by local clinical decision-making and may have varied between centres. At visit 2 (8-12 weeks later), pulmonary exacerbation status was determined based on the type of exacerbations (if any) experienced since visit 1 .

At visit 1, participants completed a socio-demographic form. At visits 1 and 2, participants completed HRQoL questionnaires and researchers completed a clinical form and measured FEV1.

\section{Socio-demographic form}

A brief self-administered, socio-demographic form was completed by participants. This included questions about age, sex, ethnicity, employment, education and current management of disease.

\section{HRQOL}

The EQ-5D and CFQ-R were used to assess HRQoL. In this study, the nondisease-specific EQ-5D preference weights were obtained from published data on a representative sample of the UK [23]. Anchors are 0 (death) and 1 (best imaginable health state). The CFQ- $\mathrm{R}$ is a disease-specific questionnaire with 12 domains that measure symptoms and HRQoL; scores range from 0 to 100 , with higher scores indicating better HRQoL (fig. S1) [18].

\section{Clinical form}

At visit 1, to collect data on healthcare utilisation associated with CF in the 12 months prior to the visit, a 1-yr retrospective chart review was conducted. In CF, patient charts' include details of both pulmonary exacerbations managed in hospital and at home. The number of pulmonary exacerbations during the previous 12 months was recorded, along with the number of pulmonary exacerbations with and without hospitalisation. The total number of days spent in hospital and the usage of $i . v$. 
antibiotics in the hospital was captured over the prior year. In addition, the usage of antibiotics at home was also recorded. Data on follow-up for hospital and home exacerbations, including number of events/visits by type of provider/specialist, was collected. Data on follow-up for hospital and home exacerbations excluded previously scheduled clinic visits, as well as telephone follow-up consultations. Data on nebulised maintenance antibiotic treatments were collected. At visit 2, data on healthcare utilisation between visit 1 and visit 2 was collected.

\section{FEV 1}

FEV1 was measured and recorded at both visits. The FEV1 \% predicted was based on age, sex and height using the calculations from KNUDSEN et al. [24].

\section{Analysis}

Descriptive statistics were calculated for demographic data and for all clinical and HRQoL outcomes collected. For patients who did not have pulmonary exacerbations at visit 1 or 2 , change in scores were calculated between visits 1 and 2 to establish the stability of the EQ-5D and the CFQ-R scores. Confidence intervals on EQ-5D utility index by visit 1 pulmonary exacerbation status were calculated based on $\beta$ distributions in our study bounded by 0 and 1 . Pearson correlations and multiple regressions were used to assess relationships between CFQ-R domain scores and EQ-5D utility index. For the multiple regressions, the EQ-5D utility index was the dependent variable and the predictor variables were the individual domains of the CFQ-R.

Resource utilisation data were decomposed into the number of pulmonary exacerbations by patient and the healthcare utilisation per pulmonary exacerbation. Visit data were pooled to better estimate the resource use per pulmonary exacerbation.

\section{RESULTS}

\section{Patient characteristics}

The demographics and spirometry of the 94 patients recruited according to baseline pulmonary exacerbation status are summarised in table 1. Among these patients, 60 had no pulmonary exacerbation, 15 had a mild pulmonary exacerbation and 19 had a severe pulmonary exacerbation.

\section{CFQ-R and EQ-5D in patients with CF and chronic P. aeruginosa}

At visit 1, CFQ-R scores reported according to pulmonary exacerbation status showed that the worse the pulmonary exacerbation status, the lower the mean CFQ-R scores for the 12 domains and the poorer the quality of life (there were slight exceptions for mild pulmonary exacerbation versus no pulmonary exacerbation, such as eating disturbance, digestion, body image and weight) (fig. 1). Mean scores by domain ranged from 42 to 85 for no pulmonary exacerbation, 55 to 82 for mild pulmonary exacerbation, and 25 to 78 for severe pulmonary exacerbation.

EQ-5D descriptive scores (fig. 2) confirm that patients with more severe pulmonary exacerbations have poorer HRQoL. EQ-5D utility index means (95\% CI, $\beta$ distribution) were 0.85 (0.80-0.89), $0.79(0.67-0.91)$ and $0.60(0.44-0.76)$ for no, mild and severe pulmonary exacerbations, respectively. The UK general population norm for age $25-34$ yrs, both sexes, is 0.93 and for age $35-44$ yrs, is 0.91 . The mean EQ-5D utility index for each pulmonary exacerbation status in our study is significantly lower than the population norm $(\mathrm{p}<0.05)$.

EQ-5D visual analogue scale scores (95\% CI, $\beta$ distribution) showed a similar pattern: mild pulmonary exacerbation 63 (53$72)$; severe pulmonary exacerbation 42 (35-48); and no pulmonary exacerbation 71 (67-75).

TABLE 1 Baseline patient demographic and clinical characteristics ${ }^{\#}$

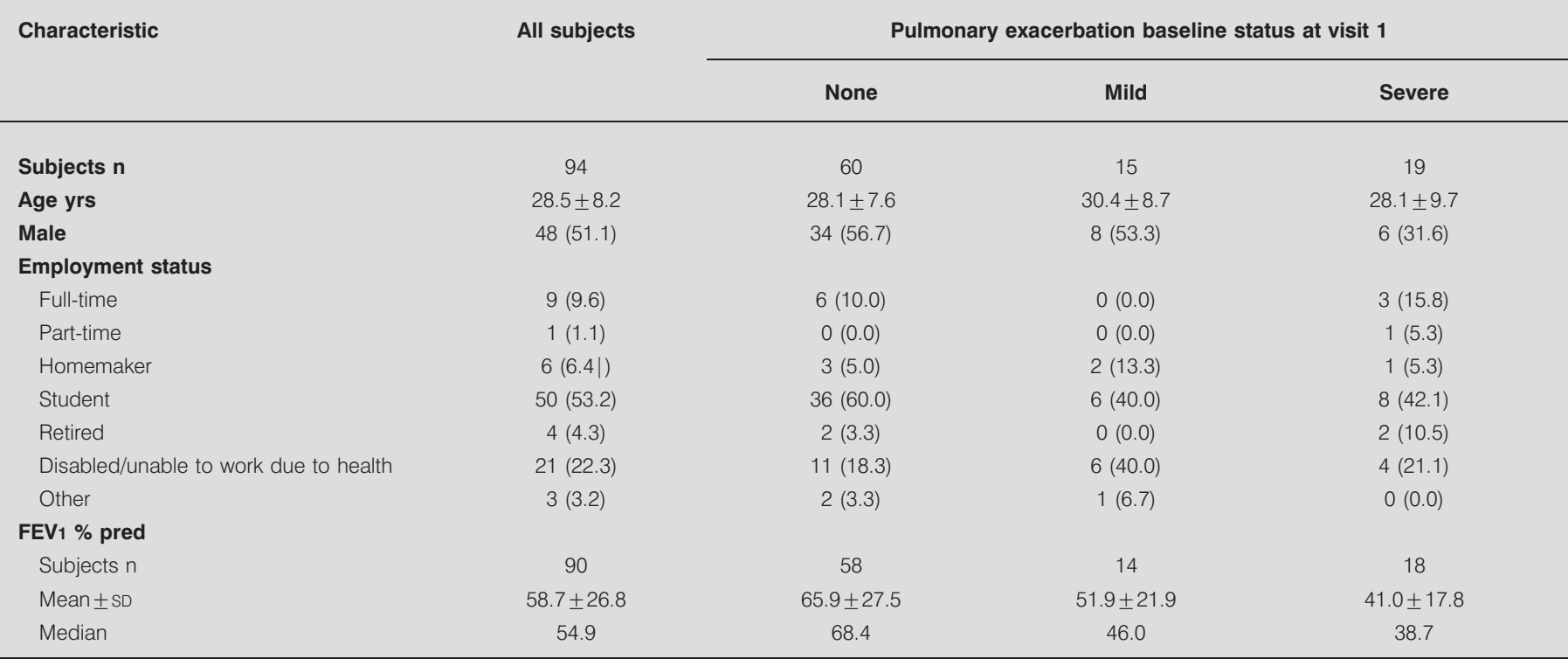

Data are presented as mean \pm SD or $n(\%)$, unless otherwise stated. FEV1: forced expiratory volume in $1 \mathrm{~s} ; \%$ pred: \% predicted. ${ }^{\#}$ : socio-demographic information was obtained using the Cystic Fibrosis Questionnaire-Revised at visit 1 unless data was missing, in which case it was imputed from other data sources. 


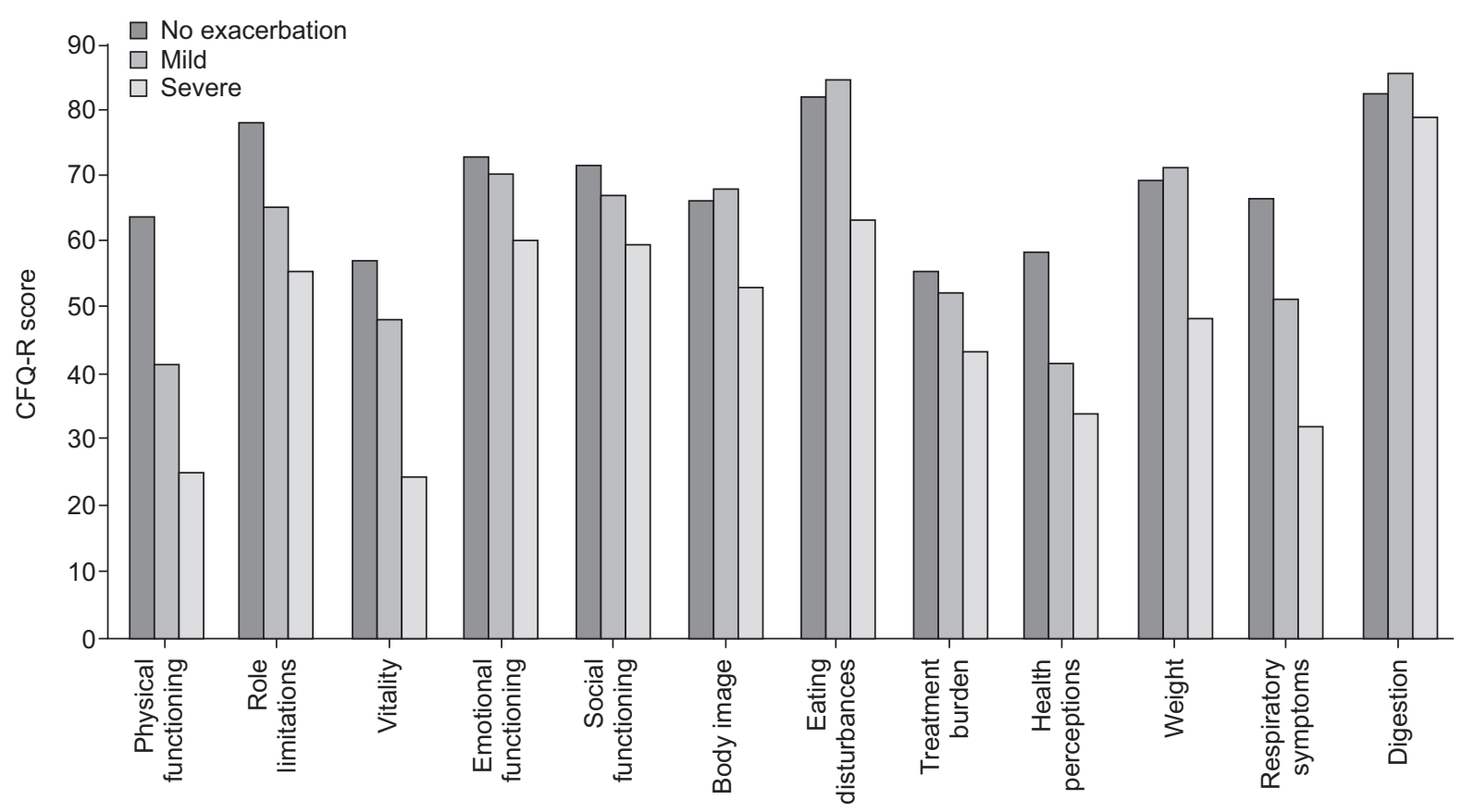

FIGURE 1. Cystic Fibrosis Questionnaire-Revised (CFQ-R) domain scores at visit 1.

For patients with no pulmonary exacerbation who had no pulmonary exacerbation at either visit 1 or at visit 2 (812 weeks later), the change in scores from visit 1 to visit 2 for the CFQ-R and EQ-5D was generally small, confirming that both instrument measurements were stable over time (figs S1 and S2). For the CFQ-R, the mean change scores were within the published minimal clinically important difference of four points [25].

\section{Relationship between CFQ-R domains and EQ-5D}

There were significant and strong relationships between domain scores of CFQ-R and EQ-5D utility index score at visit 1 and visit 2 (table 2). At visit 1, 12 domains together in a single multivariate linear regression model (not shown) explained $72 \%$ of the variance in EQ-5D $\left(\mathrm{R}^{2}=0.72\right)$ (see online supplement 2 for a five-domain model).

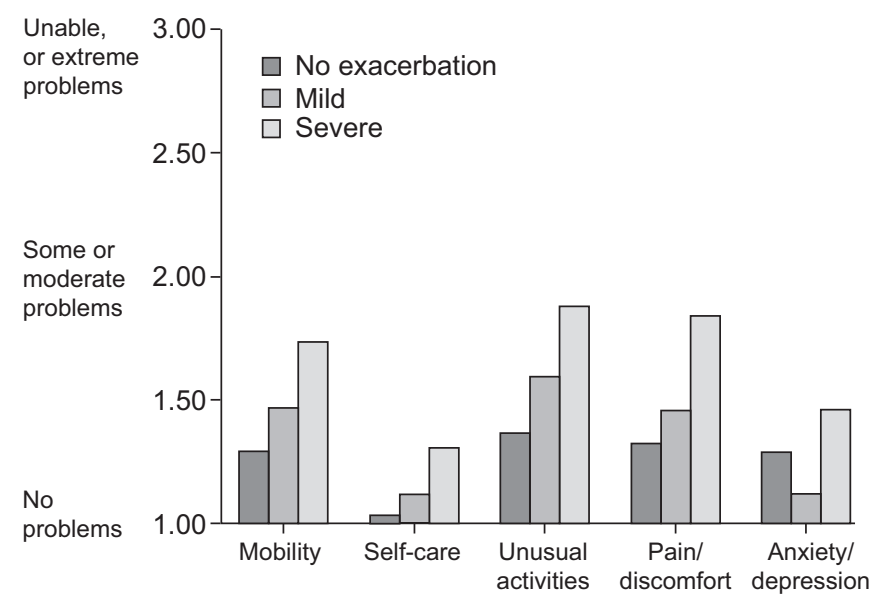

FIGURE 2. EuroQol-5D domain scores at visit 1.
Healthcare utilisation data for pulmonary exacerbations (whether treated in hospital or at home) and maintenance of inhaled antibiotic therapy in the year prior to visit 1

The mean number of pulmonary exacerbations (either requiring hospitalisation or being treated at home) in the previous year was 3.6 per patient. The mean rate of hospital pulmonary exacerbation in the previous year was 1.5 per patient and the mean rate of home-treated pulmonary exacerbations in the previous year was 2.2 per patient. Over half $(57 \%)$ of patients had at least one pulmonary exacerbation requiring hospitalisation. $83 \%$ of patients had home-treated pulmonary exacerbations, with $21 \%$ of patients having one pulmonary exacerbation and $62 \%$ having multiple pulmonary exacerbations. For the 150 hospital pulmonary exacerbations, the mean length of stay was 9.2 days per pulmonary exacerbation (table 3 ). Half of the severe pulmonary exacerbations required home i.v. antibiotics after discharge and 95\% required oral antibiotics. In the 217 home-treated pulmonary exacerbations, nearly half $(42 \%)$ of the pulmonary exacerbations required i.v. antibiotics and $71 \%$ oral antibiotics. The mean length of i.v. antibiotic treatment was 7.6 days per pulmonary exacerbation and the mean length of oral antibiotics was 10 days per pulmonary exacerbation.

The majority of patients (91\%) used inhaled antibiotic maintenance therapies and this was generally colomycin $(50 \%)$ or tobramycin $(12 \%)$ or a cyclic combination of these $(24 \%)$.

\section{DISCUSSION}

This study provides evidence that pulmonary exacerbations have an impact on HRQoL, which can be captured by diseasespecific (CFQ-R) and generic (EQ-5D) assessment tools.

The construct validity of the EQ-5D has been established in several chronic diseases; however, it has not been widely used in $\mathrm{CF}$, where the preference is to use the various diseasespecific HRQoL instruments, especially as these disease-specific 


\begin{tabular}{|c|c|c|}
\hline TABLE 2 & \multicolumn{2}{|c|}{$\begin{array}{l}\text { Relationship between domain scores Cystic } \\
\text { Fibrosis Questionnaire-Revised (CFQ-R) and } \\
\text { EuroQol-5D (EQ-5D) utility index at visit } 1\end{array}$} \\
\hline \multicolumn{2}{|c|}{ CFQ-R domain } & $\begin{array}{l}\text { Correlation between CFQ-R domain and } \\
\text { EQ-5D utility index at visit } 1^{\#}\end{array}$ \\
\hline \multicolumn{2}{|c|}{ Physical functioning } & $0.635^{\circ}$ \\
\hline \multicolumn{2}{|c|}{ Role limitations } & $0.672^{\circ}$ \\
\hline \multicolumn{2}{|c|}{ Vitality } & $0.572^{\circ}$ \\
\hline \multicolumn{2}{|c|}{ Emotional functioning } & $0.529^{\circ}$ \\
\hline \multicolumn{2}{|c|}{ Social functioning } & $0.595^{\circ}$ \\
\hline \multicolumn{2}{|c|}{ Body image } & $0.203^{*}$ \\
\hline \multicolumn{2}{|c|}{ Eating disturbances } & $0.559^{\circ}$ \\
\hline \multicolumn{2}{|c|}{ Treatment burden } & $0.438^{\circ}$ \\
\hline \multicolumn{2}{|c|}{ Health perceptions } & $0.554^{\circ}$ \\
\hline \multicolumn{2}{|c|}{ Weight } & $0.234^{*}$ \\
\hline \multicolumn{2}{|c|}{ Respiratory symptoms } & $0.545^{\circ}$ \\
\hline \multicolumn{2}{|c|}{ Digestion } & $0.478^{\circ}$ \\
\hline
\end{tabular}

Data are presented as Pearson-r. ${ }^{*}: n=91 .{ }^{*}: p<0.05 ; ": p<0.0001$.

questionnaires are accepted by the European Medicines Agency EMA as evidence of clinical benefit $[17,26]$. The EQ-5D correlated strongly with disease-specific domains of the CFQ-R. The EQ-5D utility index scores from this study provide a preference-based assessment that can be used to calculate quality-adjusted life-years for cost-effectiveness analyses of inhaled antibiotic treatments in CF. This is particularly relevant as more therapies become available for use in CF for the management of specific components of the disease. The patients in our study were representative of the UK CF population in 2008 in terms of lung function for adult centres in the UK, i.e. of 4,286 people, the mean FEV1 was $66.4 \%$ and the median proportion with P. aeruginona was $60 \%$ (UK CF Registry, Cystic Fibrosis Trust, 2011), and so the health utility data from this study are an important first step towards measuring the extent of health gain that results from healthcare interventions such as new inhaled antibiotics.

The EQ-5D in the present study allows for comparisons with other respiratory populations when stable and during an acute pulmonary exacerbation, as the dimensions were relevant to overall well-being. The detriments in health utility data seen in this adult CF population are greater than the decrements seen in paediatric CF populations; however, they are comparable to other adult respiratory populations [26-29]. There are limited data on EQ-5D decrements in utility for pulmonary exacerbations in other respiratory populations (in our investigation, data were only found in chronic obstructive pulmonary disease (COPD)). Both populations appear to experience a decrement in EQ-5D during a pulmonary exacerbation; however, the patients with CF in our study did not experience as great a decrease in EQ-5D as the COPD population [30]. In summary, the CFQ-R is likely to be most useful when exploring disease-specific components of HRQoL in CF; however, in conditions where it is important to make comparisons of HRQoL with other populations or when the aim is to calculate quality-adjusted life-years for cost-effectiveness analyses, researchers should consider using the EQ-5D.

This study provides important information on the healthcare utilisation associated with pulmonary exacerbation. The mean number of hospital i.v. treatment days for each pulmonary exacerbation was 9.2, with half of the patients continuing i.v. therapy at home. This compares well with data from the 2008 UK CF Registry Annual Data Report (which includes data for UK adult centres), i.e. 4,286 subjects with a total i.v. treatment of 24.3 days, hospital i.v. treatment of 12.1 days and home i.v. treatment of 12.1 days [31]. There is no data on the frequency of pulmonary exacerbations per year in the UK CF Registry, so our study provides important new information. Studies have identified that patients with frequent pulmonary exacerbations experienced an accelerated decline in lung function and had a greater 3-yr risk of death or transplant, and therefore warrant diligent monitoring and timely consideration for lung transplant [5].

This aggressive approach to the treatment of pulmonary exacerbations in CF is accompanied by increased rates of inpatient care costs. For example, rates of in-patient care increased significantly from $24.0 \%$ of individuals with CF in 2001 to $38.9 \%$ in $2007(\mathrm{p}<0.0001)$. Average in-patient costs varied from year to year, but also showed an increasing trend across this period [12].

\section{TABLE 3 Healthcare utilisation use per exacerbation\#}

Healthcare resource

Healthcare resource
Subjects $\mathrm{n}$
Mean hospital days
Admitted through ER \% of exacerbations
Intravenous treatment days
Oral treatment days
Post-hospital i.v. treatment at home \% of exacerbations
Post-hospital i.v. treatment days
Follow-up visits

$\begin{array}{cc}150 & 217 \\ 9.2 & \text { NA } \\ 3 & \text { NA } \\ 10.4 & 7.6^{+} \\ \text {NA } & 10 \\ 50.7 & \text { NA } \\ 4.9 & \text { NA } \\ 0.37 & 0.87\end{array}$

ER: emergency room; NA: not applicable. ${ }^{\#}$ : pooled across exacerbations reported in the first year prior to visit 1 and exacerbations reported between visit 1 and visit 2 ; ๑: excludes previously scheduled clinic visits and telephone follow-up consulations; ${ }^{+}: 42 \%$ of home exacerbations treated, for a mean of 18 days per treated exacerbation. 
Healthcare utilisation data provide an important estimate of the burden of CF treatment on healthcare resources. This is essential for progression planning and forecasting staffing requirements, especially with regard to key members of the multidisciplinary team, e.g. nurses and physiotherapists, who provide intensive daily treatment during pulmonary exacerbation. The effect on reducing pulmonary exacerbation is an efficacy criterion accepted by regulatory agencies as evidence of treatment benefit, and pulmonary exacerbations have been co-primary outcomes for many therapies approved for CF. Therefore, the healthcare utilisation data from this study provides important information to facilitate accurate sample size calculations for future clinical trials.

The majority of patients used inhaled antibiotic maintenance therapies and this was generally colomycin or tobramycin. Data from the 2008 UK CF Registry Annual Data Report show that this is representative of the use of inhaled antibiotics in other UK CF centres (20\% of patients on Tobi ${ }_{\circledast}$ or Bramitob ${ }_{\circledR}$; $64 \%$ on colomycin/promixin; and $5 \%$ on gentamicin). The higher usage of colistin is likely to be explained by UK guidelines, which indicate that colistin should be used initially for treatment of chronic $P$. aeruginosa and, thereafter, tobramycin should be considered as a second-line treatment approach in nonresponders. Unlike the rest of Europe, in the UK, the funding for inhaled antibiotic therapy is dictated by region. It is very important to increase the evidence-base to rationalise the use of these and new inhaled therapies and analyse these treatments to provide data on their costeffectiveness.

Although research co-ordinators were encouraged to use symptom-based criteria to define whether the patient had a pulmonary exacerbation or not, the actual categorisation of pulmonary exacerbations in this study was based on the site of treatment, i.e. the pulmonary exacerbation was defined as severe if it was treated at the hospital and mild if it was treated at home (regardless of whether the patient received i.v. antibiotics or oral therapy). There is no agreed definition for a pulmonary exacerbation and there is also no agreement on what constitutes a severe or mild pulmonary exacerbation. Although it is recognised that there are likely to be differences between the sites around decision-making for home versus hospital i.v. therapy, in our study, we assumed that patients hospitalised for i.v. therapy had severe pulmonary exacerbations requiring daily intensive treatment and medical monitoring. The study does give insights into the health status associated with pulmonary exacerbations.

The findings are also limited by the small sample size and our inability to achieve our goal of 30 patients per pulmonary exacerbation status within the recruitment timeframe. We were also unable to use the recognised definition for chronic $P$. aeruginosa [32], and had to assume that investigators followed inclusion criteria and recruited patients who had been diagnosed with chronic $P$. aeruginosa according to local criteria. To verify this, we explored patients' use of maintenance inhaled/oral antibiotics, which confirmed that all patients were likely to have chronic infection. Another limitation relates to the resource utilisation, which was collected through retrospective chart review. Only data in the charts could be included in this study and missing data (e.g. oral antibiotics courses initiated by the patient's general practitioner) is a common problem with these types of studies. Despite these limitations, which are common to many studies of this design, the data collected from the patients enrolled in this observational study contribute new knowledge to the association between EQ5D and CFQ-R, decrements associated with pulmonary exacerbations, and resource use for patients with CF.

This study has shown that patients with CF and chronic $P$. aeruginosa infection have frequent pulmonary exacerbations. As pulmonary exacerbation status worsens, patients experience worse HRQoL as measured by CFQ-R and EQ-5D. There is significant healthcare utilisation associated with pulmonary exacerbations in CF. Therapies aimed at preventing and treating pulmonary exacerbations are important to maintain health status and lung function in CF. This study showed that the EQ-5D utility index is explained by changes in CFQ-R and, therefore, our study provides credibility for the use of EQ-5D for economic evaluation of healthcare interventions in CF.

\section{SUPPORT STATEMENT}

This study is sponsored by Novartis (Basel, Switzerland).

\section{STATEMENT OF INTEREST}

Statements of interest for all authors and the study itself can be found at www.erj.ersjournals.com/site $/ \mathrm{misc} /$ statements.xhtml

\section{ACKNOWLEDGEMENTS}

The authors thanks other investigators/research co-ordinators: S. Martin (Belfast Health and Social Care Trust City Hospital, Belfast, UK); F. Lloyd (Birmingham Heart of England NHS Trust, Birmingham, UK); S. Patel (Bristol University Hospital, Bristol, UK); L. Bennett and L. Priestly (Oxford Radcliffe Hospital, Oxford, UK); and L. Kuitert and C. Dawson (London Barts, London, UK).

\section{REFERENCES}

1 McCormick J, Green MW, Mehta G, et al. Demographics of the UK cystic fibrosis population: implications for neonatal screening. Eur J Hum Genet 2002; 10: 583-590.

2 Dodge JA, Lewis PA, Stanton M, et al. Cystic fibrosis mortality and survival in the UK: 1947-2003. Eur Respir J 2007; 29: 522-526.

3 Razvi S, Quittell L, Sewall A, et al. Respiratory microbiology of patients with cystic fibrosis in the United States, 1995 to 2005. Chest 2009; 136: 1554-1560.

4 Liou TG, Adler FR, Fitzsimmons SC, et al. Predictive 5-year survivorship model of cystic fibrosis. Am J Epidemiol 2001; 153: 345-352.

5 Boer K, Vandemheen KL, Tullis E, et al. Exacerbation frequency and clinical outcomes in adult patients with cystic fibrosis. Thorax 2011; 66: 680-685.

6 Flume PA, Mogayzel PJ, Robinson KA, et al. Cystic fibrosis pulmonary guidelines: treatment of pulmonary exacerbations. Am J Respir Crit Care Med 2009; 180: 802-808.

7 Flume PA, O'Sullivan BP, Robinson KA, et al. Cystic fibrosis pulmonary guidelines: chronic medications for maintenance of lung health. Am J Respir Crit Care Med 2007; 176: 957-969.

8 Ramsey BW, Pepe MS, Quan JM, et al. Intermittent administration of inhaled tobramycin in patients with cystic fibrosis. N Engl J Med 1999; 340: 23-30.

9 Konstan MW, Geller DE, Minić P, et al. Tobramycin inhalation powder for P. aeruginosa infection in cystic fibrosis: the EVOLVE trial. Pediatr Pulmonol 2010; 46: 230-238. 
10 Konstan MW, Flume PA, Kappler M, et al. Safety, efficacy and convenience of tobramycin inhalation powder in cystic fibrosis patients: the EAGER trial. J Cyst Fibros 2011; 10: 54-61.

11 Oermann CM, Retsch-Bogart GZ, Quittner AL, et al. An 18-month study of the safety and efficacy of repeated courses of inhaled aztreonam lysine in cystic fibrosis. Pediatr Pulmonol 2010; 45: 1121-1134.

12 Briesacher BA, Quittner AL, Fouavzi $\mathrm{H}$, et al. Nationwide trends in the medical care costs of privately insured patients with cystic fibrosis (CF), 2001-2007. Pediatr Pulmonol 2011; 46: 770-776.

13 Ouyang L, Grosse SD, Amendah DD, et al. Healthcare expenditures for privately insured people with cystic fibrosis. Pediatr Pulmonol 2009; 44: 989-996.

14 Weiner, JR Toy EL, Sacco P, et al. Costs, quality of life and treatment compliance associated with antibiotic therapies in patients with cystic fibrosis: a review of the literature. Expert Opin Pharmacother 2008; 9: 751-766.

15 Britto MT, Kotagal UR, Hornung RW, et al. Impact of recent pulmonary exacerbations on quality of life in patients with cystic fibrosis. Chest 2002; 121: 64-72.

16 Dobbin CJ, Bartlett D, Melehan K, et al. The effect of infective exacerbations on sleep and neurobehavioural function in cystic fibrosis. Am J Respir Crit Care Med 2005; 172: 99-104.

17 European Medicines Agency, Committee for Medicinal Products for Human Use. Guideline on the Clinical Development of Medicinal Products for the Treatment of Cystic Fibrosis. www. ema.europa.eu/docs/en_GB/document_library/Scientific_guideline/ 2009/12/WC500017055.pdf Date last updated: May 12, 2011. Date last accessed: December 20, 2011.

18 Quittner A, Buu A, Messer MA, et al. Development and validation of the cystic fibrosis questionnaire in the United States: a health-related quality-of-life measure for cystic fibrosis. Chest 2005; 128: 2347-2354.

19 Rabin R, de Charro F. EQ-5D a measure of health status from the EuroQol group. Ann Med 2001; 33: 337-343.

20 The EuroQol Group. EuroQol - a new facility for the measurement of health-related quality of life. Health Policy 1990; 16: 199-208.

21 Kind P. The EuroQol instrument: an index of health-related quality of life. In: Spilker B, ed. Quality of Life and Pharmacoeconomics in Clinical Trials. 2nd Edn. Philadelphia, LippincottRaven Publishers, 1996; pp. 191-202.
22 Goss $\mathrm{CH}$, Burns JL. Exacerbations in cystic fibrosis. 1: epidemiology and pathogenesis. Thorax 2007; 62: 360-367.

23 MVH Group. The measurement and valuation of health. Final report on the modelling of valuation tariffs. York, Centre for Health Economics, 1995.

24 Knudson RJ, Lebowitz MD, Holberg CJ, et al. Changes in the normal maximal expiratory flow-volume curve with growth and aging. Am Rev Respir Dis 1983; 127: 725-734.

25 Quittner AL, Modi AC, Wainwright C, et al. Determination of the minimal clinically important difference scores for the cystic fibrosis questionnaire-revised respiratory symptom scale in two populations of patients with cystic fibrosis and chronic Pseudomonas aeruginosa airway infection. Chest 2009; 135: 1610-1618.

26 Eidt-Koch D, Mittendorf T, Greiner W. Cross-sectional validity of the EQ-5D-Y as a generic health outcome instrument in children and adolescents with cystic fibrosis in Germany. BMC Pediatrics 2009; 9: 2431-2439.

27 Johnson JA, Connolly M, Zuberbuhler $\mathrm{P}$, et al. Health-related quality of life for adults with cystic fibrosis: a regression approach to assessing impact of recombinant human DNase. Pharmacotherapy 2000; 20: 1167-1174.

28 Polley L, Yaman N, Heaney L, et al. Impact of cough across different chronic respiratory diseases. Comparison of two coughspecific health-related quality of life questionnaires. Chest 2008; 134: 295-302.

29 Rutten-van Molken M, Oostenbrink JB, Tashkin DP, et al. Does quality of life of COPD patients as measured by the generic EuroQol five-dimension questionnaire differentiate between COPD severity stages? Chest 2006; 130: 1117-1128.

30 O'Reilly JF, Williams AE, Rice L. Health status impairment and costs associated with COPD exacerbation managed in hospital. Int J Clin Pract 2007; 61: 1112-1120.

31 UK CF Registry. Annual Data Report 2008. http://www.cftrust. org.uk/aboutcf/publications/cfregistryreports/UK_CF_RegistryAnnual_Data_Report_2008.pdf Date last accessed. October 12, 2012.

32 Pressler T, Bohmova C, Conway S, et al. Chronic Pseudomonas aeruginosa infection definition: EuroCareCF Working Group report. J Cyst Fibros 2011; 10: Suppl. 2, S75-S78. 